\title{
Alpha-Skew-Logistic Distribution
}

\author{
Partha Jyoti Hazarika ${ }^{a}$ and Subrata Chakraborty ${ }^{b}$ \\ ${ }^{a}$ Department of Mathematics, NIT, Meghalaya, India \\ ${ }^{\mathrm{b}}$ Department of Statistics, Dibrugarh University, Dibrugarh-786 004, Assam, India
}

\begin{abstract}
Alpha-skew-Logistic distribution is introduced following the same methodology as those of Alphaskew-normal distribution (Elal-Olivero, 2010) and Alpha-skew-Laplace distributions (Harandi and Alamatsaz, 2013). Cumulative distribution function (cdf), moment generating function (mgf), moments, skewness and kurtosis of the new distribution is studied. Some related distributions are also investigated. Parameter estimation by method of moment and maximum likelihood are discussed. Closeness of the proposed distribution with alpha-skew-normal distribution is studied. The suitability of the proposed distribution is tested by conducting data fitting experiment and comparing the values of log likelihood, AIC, BIC. Likelihood ratio test is used for discriminating between Alpha-skew-Logistic and logistic distributions.
\end{abstract}

Mathematics Subject Classification: 60E05; 62E15; 62H10

Keywords: Alpha-Skew normal distribution; Multimodal distribution; Alpha-skew normal; Alpha-skew Laplace; Kullback Leibler (KL) distance.

\section{Introduction}

Azzalini (1985) first introduced the path breaking skew-normal distribution, which is nothing but a natural extension of normal distribution derived by adding an additional asymmetry parameter and the probability density function (pdf) of the same is given by

$$
f_{Z}(z ; \lambda)=2 \phi(z) \Phi(\lambda z) ; \quad z \in R, \lambda \in R
$$

where $\phi$ and $\Phi$ are the pdf and cdf of standard normal distribution respectively. Here, $\lambda$ is the asymmetry parameter. Following Azzalini's (1985) seminal paper, a lot of research work has so far been carried out to present different skew normal distributions derived from the underlying symmetric one to model asymmetric behavior of empirical data suitable under different situations (For a complete survey on univariate skew normal distributions see Chakraborty and Hazarika (2011)).

Huang and Chen (2007), proposed the general formula for the construction of skew-symmetric distributions starting from a symmetric (about 0 ) pdf $h($.) by introducing the concept of skew function $G($.$) , a$ Lebesgue measurable function such that, $0 \leq G(z) \leq 1$ and $G(z)+G(-z)=1 ; Z \in R$, almost everywhere. A random variable $Z$ is said to be skew symmetric if the pdf is of the following form

$$
f_{Z}(z)=2 h(z) G(z) ; z \in R
$$

Choosing an appropriate skew function $G(z)$ in the equation (2) one can construct different skew distributions with both unimodal and multimodal behavior. Chakraborty et al., [2012, 2014a, and 2014b] introduced new skew logistic, skew Laplace and skew normal distributions which are multimodal with peaks occurring at intervals by considering a periodic skew function involving trigonometric "sine" function and studied their different properties including parameter estimation and data fitting. Alpha-skew-normal distribution was introduced by Elal-Olivero (2010) as a new class of skew normal distribution that includes both unimodal as well as bimodal normal distributions. Using exactly the similar approach Harandi and Alamatsaz (2013) investigated a class of alpha-skew-Laplace distributions and very recently Gui (2014) introduced alpha skew normal slash distribution and studied its properties.

The logistic distribution which very often is seen as a competitor of normal and Laplace distribution is an important probability distribution which attracted wide attention from both theoretical and application point of view (see Johnson et al., 2004 and Balakrishnan, 1992). Again, like other skewed distributions, the skewed version of the logistic distribution also has wide range of applications in real life (for detail see Wahed and Ali (2001); Nadarajah and Kotz, (2006 and 2007); Nadarajah (2009); Chakraborty et al., (2012)). In the present study we have borrowed the idea from Elal-Olivero (2010) and Harandi and Alamatsaz (2013) to propose and investigate an alpha-skew-logistic distribution which generalizes the logistic distribution and flexible enough to adequately model both unimodal as well as bimodal data in the presence of positive or negative skewness. 


\section{Alpha-Skew-Logistic Distribution}

In this section we introduce the proposed alpha skew logistic distribution and investigate its shape and mode numerically.

Definition 1. If an r.v. $Z$ has the density function

$$
f_{Z}(z ; \alpha)=\frac{3\left\{(1-\alpha z)^{2}+1\right\} \exp (-z)}{\left\{6+\left(\alpha^{2} \pi^{2}\right)\right\}(1+\exp (-z))^{2}} ; z \in R, \alpha \in R
$$

where, then we say $Z$ is an alpha-skew-logistic r.v. with parameter $\alpha$ and denote it by $Z \sim \operatorname{ASLG}(\alpha)$.

\section{Particular cases:}

i. for $\alpha=0$, we get the standard Logistic distribution is given by

$$
f_{Z}(z)=\frac{\exp (-z)}{(1+\exp (-z))^{2}} ; \quad z \in R
$$

and is denoted by $Z \sim \mathrm{L}(0,1)$.

ii. If $\alpha \rightarrow \pm \infty$ the we get a bimodal logistic (BLG) distribution given by

$$
f_{Z}(z)=\frac{3 z^{2} \exp (-z)}{\pi^{2}(1+\exp (-z))^{2}} ; \quad z \in R
$$

and is denoted by $Z \stackrel{d}{\longrightarrow}$ BLG

iii. If $Z \sim \operatorname{ASLG}(\alpha)$ then $-Z \sim \operatorname{ASLG}(-\alpha)$.

\subsection{Plots of the pdf}

The ASLG pdf for different choices of the parameter $\alpha$ are plotted in figure 1 .
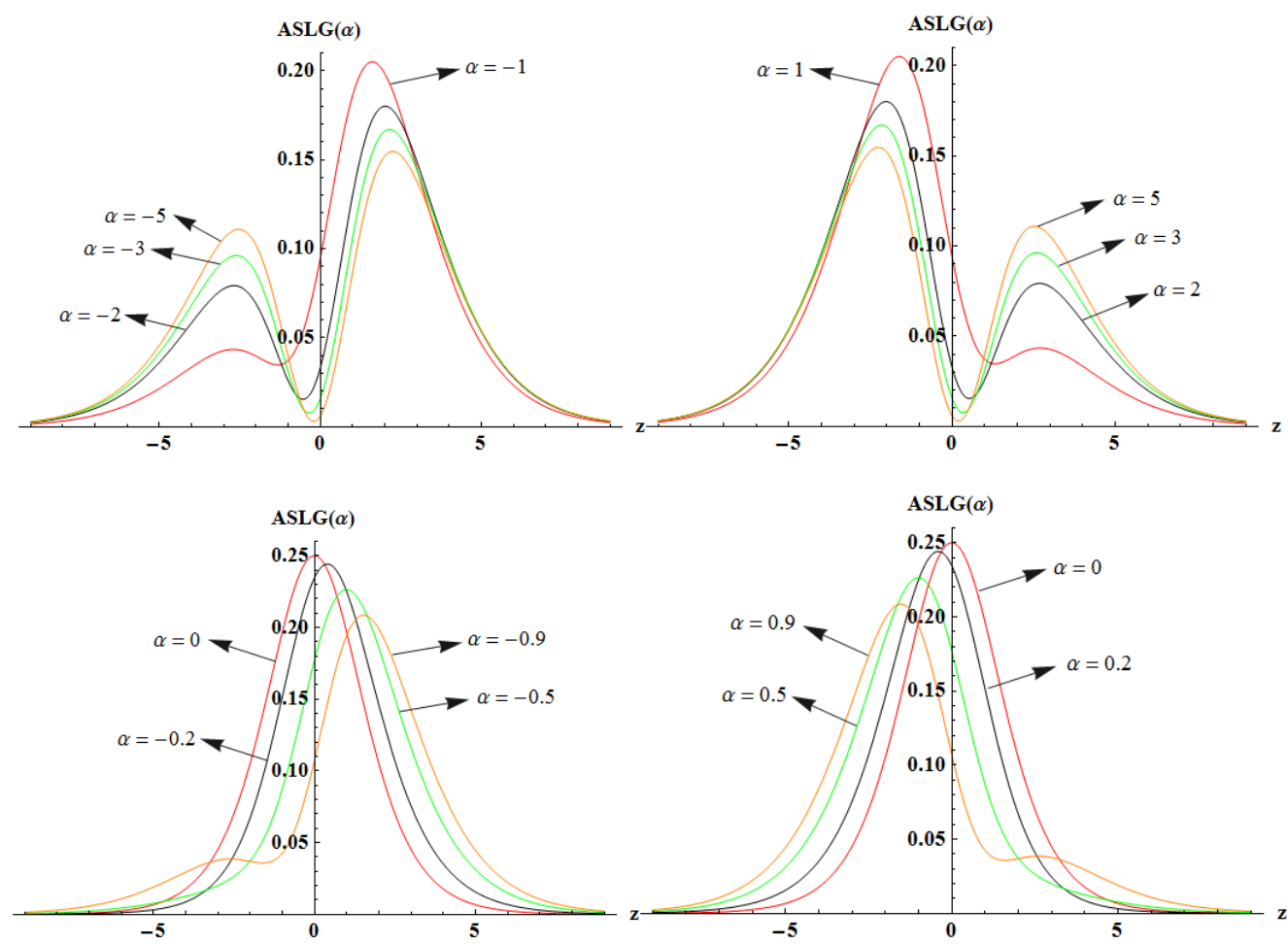

Figure 1. Plots of pdf of $\operatorname{ASLG}(\alpha)$

From the figures it can be seen that the distribution has at most two modes (see also section 2.2). Also the smaller of the two peaks when present appears in the right (left) of the higher one depending on the value of $\alpha<(>) 0$.

\subsection{Mode of ASLG}

Here we numerically verify the fact that ASLG has at most two modes.

The first differentiation of the pdf $f_{Z}(z ; \alpha)$ of $\operatorname{ASLG}(\alpha)$ with respect to $z$ is given by 


$$
D f_{Z}(z ; \alpha)=\frac{3 \exp (z)[\alpha\{\exp (z)(z(2-\alpha(z-2))-2)+z(\alpha(z+2)-2)-2\}-2 \exp (z)+2]}{\left(6+\alpha^{2} \pi^{2}\right)(\exp (z)+1)^{3}}
$$

In order to check that $\operatorname{ASLG}(\alpha)$ has at most two modes, the contour of the equation $D f_{Z}(z ; \alpha)=0$ is drawn in figure 2. It can be seen that there is at most three zeros of $D f_{Z}(z ; \alpha)$ which establishes that $\operatorname{ASLG}(\alpha)$ has at most two modes. Note that for $-0.8<\alpha<0.8 \operatorname{ASLG}(\alpha)$ remains unimodal (see the second plot in figure 2).
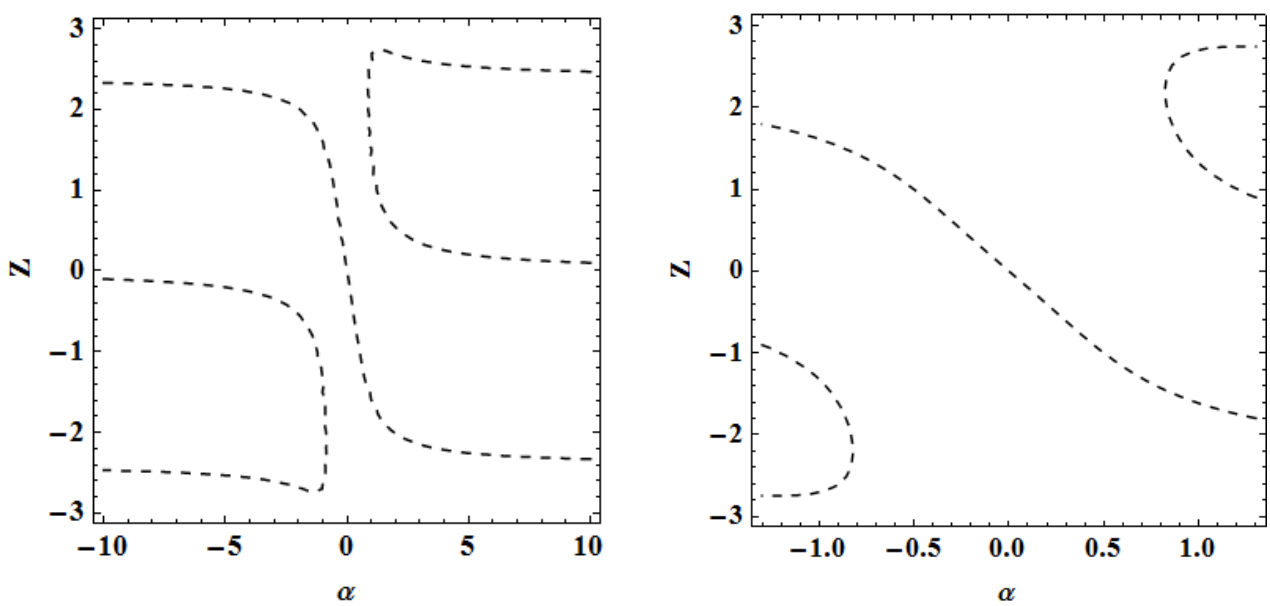

Figure 2. Contour plots of the equation $D f_{Z}(z ; \alpha)=0$

\section{Distributional Properties}

\subsection{Cumulative Distribution Function (cdf)}

Theorem 1. The cdf of $\operatorname{ASLG}(\alpha)$ is given by

$F_{Z}(z ; \alpha)=\frac{3}{\left(6+\alpha^{2} \pi^{2}\right)}\left(\frac{(1-\alpha z)^{2}+1}{1+\exp (-z)}+2 \alpha(1-\alpha z) \log \{1+\exp (z)\}-2 \alpha^{2} \operatorname{Li}_{2}[-\exp (\mathrm{z})]\right)$

Proof: $F_{Z}(z ; \alpha)=\frac{3}{\left(6+\alpha^{2} \pi^{2}\right)} \int_{-\infty}^{z} \frac{\left\{(1-\alpha z)^{2}+1\right\} \exp (-z)}{(1+\exp (-z))^{2}} d z$

$$
\begin{aligned}
& =\frac{3}{\left(6+\alpha^{2} \pi^{2}\right)}\left(\int_{-\infty}^{z} \frac{2 \exp (-z)}{(1+\exp (-z))^{2}} d z-2 \alpha \int_{-\infty}^{z} \frac{z \exp (-z)}{(1+\exp (-z))^{2}} d z+\alpha^{2} \int_{-\infty}^{z} \frac{z^{2} \exp (-z)}{(1+\exp (-z))^{2}} d z\right) \\
& =\frac{3}{\left(6+\alpha^{2} \pi^{2}\right)}\left(\frac{2}{1+\exp (-z)}-\frac{2 \alpha z}{1+\exp (-z)}+2 \alpha \log (1+\exp (z))\right. \\
& \left.\quad+\frac{\alpha^{2} z^{2}}{1+\exp (-z)}-2 \alpha^{2} z \log (1+\exp (z))-2 \alpha^{2} \mathrm{Li}_{2}[-\exp (\mathrm{z})]\right) \\
& =\frac{3}{\left(6+\alpha^{2} \pi^{2}\right)}\left(\frac{(1-\alpha z)^{2}+1}{1+\exp (-z)}+2 \alpha(1-\alpha z) \log \{1+\exp (z)\}-2 \alpha^{2} \mathrm{Li}_{2}[-\exp (\mathrm{z})]\right) .
\end{aligned}
$$

Where $\operatorname{Li}_{\mathrm{n}}(z)=\sum_{k=1}^{\infty} \frac{z^{k}}{k^{n}}$ is the poly-logarithm function (see Prudnikov et al. (1986)).

The cdf is plotted in figure 3 for studying variation in its shape with respect to the parameter $\alpha$.

Corollary 1. In particular by taking limit $\alpha \rightarrow+\infty$ or $-\infty$ the cdf of BLG distribution in equation (5) is easily obtained from (7) as

$$
\frac{3 z\left\{\exp (z)-2\left(1+\exp (z) \log ((1+\exp (z))\}-6\left((1+\exp (z)) \mathrm{Li}_{2}[-\exp (\mathrm{z})]\right.\right.\right.}{(1+\exp (z)) \pi^{2}}
$$




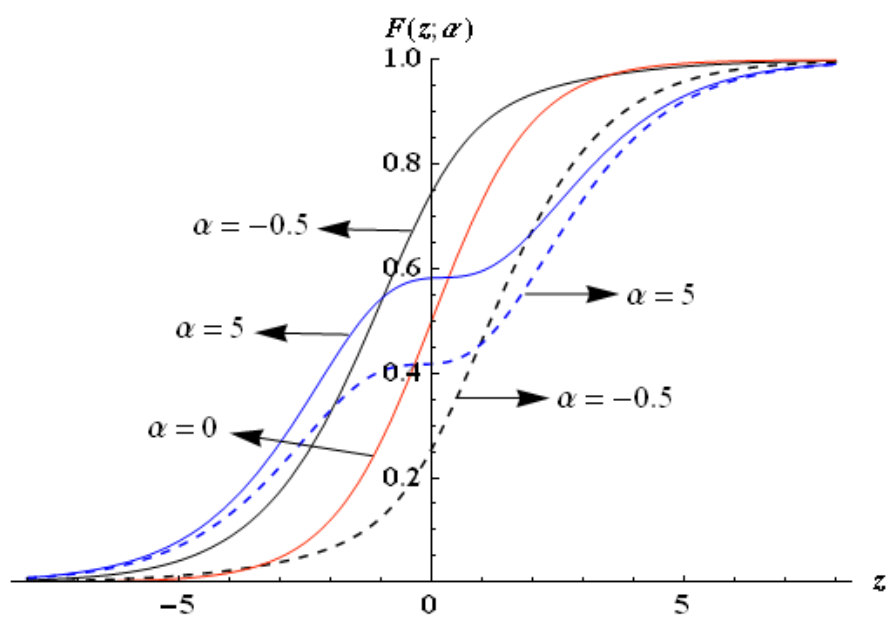

Figure 3. Plots of cdf of $\operatorname{ASLG}(\alpha)$

\subsection{Moment Generating Function (mgf) and Moments}

3.2.1 Moment Generating Function (mgf)

Theorem 2. The mgf of $\operatorname{ASLG}(\alpha)$ is given by

$M_{Z}(t)=\frac{6 M_{X}(t)}{t\left(6+\alpha^{2} \pi^{2}\right)}\left(t-\alpha\{1-\pi t \cot (\pi t)\}+\frac{\alpha^{2}\left\{M_{X}(t)\right\}^{2}}{4 \pi t^{2}}\{\pi t\{3+\cos (2 \pi t)\}-2 \sin (2 \pi t)\}\right) ;-1<t<1$,

where $X \sim \mathrm{L}(0,1)$ and $M_{X}(t)=B(1+t, 1-t)=\Gamma(1+t) \Gamma(1-t)$ is the mgf of $\mathrm{L}(0,1)$.

Proof:

$$
\begin{aligned}
M_{Z}(t) & =\frac{3}{\left(6+\alpha^{2} \pi^{2}\right)} \int_{-\infty}^{+\infty} \exp (t z) \frac{\left\{(1-\alpha z)^{2}+1\right\} \exp (-z)}{(1+\exp (-z))^{2}} d z \\
& =\frac{3}{\left(6+\alpha^{2} \pi^{2}\right)}\left(\int_{-\infty}^{+\infty} \frac{2 \exp (t z) \exp (-z)}{(1+\exp (-z))^{2}} d z-2 \alpha \int_{-\infty}^{+\infty} \frac{z \exp (t z) \exp (-z)}{(1+\exp (-z))^{2}} d z+\right. \\
& =\frac{3}{\left(6+\alpha^{2} \pi^{2}\right)}\left(2 M_{X} \int_{-\infty}^{+\infty} \frac{z^{2} \exp (t z) \exp (-z)}{(1+\exp (-z))^{2}} d z\right) \\
\frac{2 \alpha M_{X}(t)}{t}\{1-\pi t \cot (\pi t)\}+ & \left.\frac{\alpha^{2}\left\{M_{X}(t)\right\}^{3}}{2 \pi t^{3}}\{\pi t\{3+\cos (2 \pi t)\}-2 \sin (2 \pi t)\}\right)
\end{aligned}
$$

The result follows on simplification.

Corollary 2. In particular, by taking limit as $\alpha \rightarrow+\infty$ or $-\infty$ of the $M_{Z}(t)$ in equation (8), we get the mgf of BLG distribution as

$$
\frac{3\left\{M_{X}(t)\right\}^{3}}{2(\pi t)^{3}}\{\pi t\{3+\cos (2 \pi t)\}-2 \sin (2 \pi t)\}
$$

Remark 1. Alternative expression for the mgf of $\operatorname{ASLG}(\alpha)$ can be obtained in terms of gamma function and its derivatives as

$$
\begin{array}{r}
\frac{3}{\left(6+\alpha^{2} \pi^{2}\right)}\left[2 \Gamma(1+t) \Gamma(1-t)-2 \alpha\left\{\Gamma^{\prime}(1+t) \Gamma(1-t)-\Gamma(1+t) \Gamma^{\prime}(1-t)\right\}\right. \\
\left.+\alpha^{2}\left\{\Gamma^{\prime \prime}(1+t) \Gamma(1-t)+\Gamma^{\prime}(1+t) \Gamma^{\prime \prime}(1-t)\right\}\right]
\end{array}
$$

Theorem 3. The $k^{\text {th }}$ order moment of $\operatorname{ASLG}(\alpha)$ distribution is given by 
$\mathrm{E}\left(Z^{k}\right)=\frac{6}{\left(6+\alpha^{2} \pi^{2}\right)}\left\{\begin{array}{l}2\left(1-\frac{1}{2^{k-1}}\right) \Gamma(k+1) \varsigma(k)+\alpha^{2}\left(1-\frac{1}{2^{k+1}}\right) \Gamma(k+3) \varsigma(k+2) ; \text { if } k \text { even } \\ -2 \alpha\left(1-\frac{1}{2^{k}}\right) \Gamma(k+2) \varsigma(k+1) ; \text { if } k \text { odd }\end{array}\right.$

where $\varsigma(s)=\sum_{j}^{\infty} j^{-s}$ is the Riemann zeta functions and $\Gamma(s)=\int_{0}^{\infty} t^{s-1} \exp (-t) d t$.

Proof: Recall that, for $X \sim \mathrm{L}(0,1)$ i.e. for the standard logistic random variable $X$

$$
\mathrm{E}\left(X^{k}\right)=\left\{\begin{array}{ll}
2\left(1-\left(1 / 2^{k-1}\right)\right) \Gamma(k+1) \varsigma(k) ; & \text { if } k \text { is even } \\
0 ; & \text { if } k \text { is odd. }
\end{array} \quad\right. \text { (Balakrishnan, 1992) }
$$

Now, $\quad \mathrm{E}\left(Z^{k}\right)=\int_{-\infty}^{\infty} z^{k} f(z ; \alpha) d z=\frac{3}{\left(6+\alpha^{2} \pi^{2}\right)} \int_{-\infty}^{\infty} z^{k}\left(2-2 \alpha z+\alpha^{2} z^{2}\right) \frac{\exp (-z)}{(1+\exp (-z))^{2}} d z$

$$
=\frac{3}{\left(6+\alpha^{2} \pi^{2}\right)}\left[\int_{-\infty}^{\infty} \frac{2 z^{k} \exp (-z)}{(1+\exp (-z))^{2}} d z-\int_{-\infty}^{\infty} \frac{2 \alpha z^{k+1} \exp (-z)}{(1+\exp (-z))^{2}} d z+\int_{-\infty}^{\infty} \frac{\alpha^{2} z^{k+2} \exp (-z)}{(1+\exp (-z))^{2}} d z\right]
$$

Case I. when $k$ is even

Case II. when $k$ is odd

$$
\begin{aligned}
\mathrm{E}\left(Z^{k}\right) & =\frac{3}{\left(6+\alpha^{2} \pi^{2}\right)}\left[\int_{-\infty}^{\infty} \frac{2 z^{k} \exp (-z)}{(1+\exp (-z))^{2}} d z+\int_{-\infty}^{\infty} \frac{\alpha^{2} z^{k+2} \exp (-z)}{(1+\exp (-z))^{2}} d z\right] \\
& =\frac{6}{\left(6+\alpha^{2} \pi^{2}\right)}\left[2\left(1-\frac{1}{2^{k-1}}\right) \Gamma(k+1) \varsigma(k)+\alpha^{2}\left(1-\frac{1}{2^{k+1}}\right) \Gamma(k+3) \varsigma(k+2)\right]
\end{aligned}
$$

$$
\begin{aligned}
\mathrm{E}\left(Z^{k}\right) & =\frac{3}{\left(6+\alpha^{2} \pi^{2}\right)}\left[-\int_{-\infty}^{\infty} \frac{2 \alpha z^{k+1} \exp (-z)}{(1+\exp (-z))^{2}} d z\right] \\
& =\frac{6}{\left(6+\alpha^{2} \pi^{2}\right)}\left[-2 \alpha\left(1-\frac{1}{2^{k}}\right) \Gamma(k+2) \varsigma(k+1)\right]
\end{aligned}
$$

The result (10) follows immediately.

\subsubsection{Moments}

From (10) using the following particular values of the Riemann zeta functions, $\varsigma(2)=\pi^{2} / 6, \varsigma(4)=\pi^{4} / 90$ and $\varsigma(6)=\pi^{9} / 945$ (Prudnikov et al. 1986), we get

$$
\mathrm{E}(Z)=\mu_{1}^{\prime}=\frac{-2 \alpha \pi^{2}}{6+\alpha^{2} \pi^{2}},
$$

Which is proportional to variance of standard logistic distribution and is positive (or negative) if $\alpha<(>) 0$.

$$
\begin{aligned}
& \mathrm{E}\left(Z^{2}\right)=\mu_{2}^{\prime}=\frac{10 \pi^{2}+7 \alpha^{2} \pi^{4}}{5\left(6+\alpha^{2} \pi^{2}\right)} \\
& \mathrm{E}\left(Z^{3}\right)=\mu_{3}^{\prime}=\frac{-14 \alpha \pi^{4}}{5\left(6+\alpha^{2} \pi^{2}\right)}, \text { is positive (or negative) if depending on whether } \alpha<(>) 0 . \\
& \mathrm{E}\left(Z^{4}\right)=\mu_{4}^{\prime}=\frac{\pi^{4}\left(98+155 \alpha^{2} \pi^{2}\right)}{35\left(6+\alpha^{2} \pi^{2}\right)} \\
& \operatorname{Var}(Z)=\mu_{2}=\frac{\pi^{2}\left(60+32 \alpha^{2} \pi^{2}+7 \alpha^{4} \pi^{4}\right)}{5\left(6+\alpha^{2} \pi^{2}\right)^{2}}=\frac{\pi^{2}}{3}\left\{\frac{\left(180+96 \alpha^{2} \pi^{2}+21 \alpha^{4} \pi^{4}\right)}{15\left(6+\alpha^{2} \pi^{2}\right)^{2}}\right\}
\end{aligned}
$$

Since, $\frac{\left(180+96 \alpha^{2} \pi^{2}+21 \alpha^{4} \pi^{4}\right)}{15\left(6+\alpha^{2} \pi^{2}\right)^{2}} \geq 1$ therefore, variance of $\operatorname{ASLG}(\alpha)$ is greater than or equal to that of $L(0,1)$. 
Remark 2. From the compact formulas of the moments obtained above we can see that $\mu_{3}^{\prime} / \mu_{1}^{\prime}=1.4 \pi^{2}$ $=13.8286$, which means that the third moment is increasing (decreasing) function of the mean according as the parameter $\alpha<(>) 0$. Further, $\alpha=0.217 \frac{\mu_{3}^{\prime}}{\left(\mu_{1}^{\prime}\right)^{2}}=\frac{0.3001}{\mu_{1}^{\prime}}$ and hence the pdf of in equation (3) can be written in terms of $\mu_{1}^{\prime}$ as

$$
f_{Z}\left(z ; \mu_{1}^{\prime}\right)=\frac{3\left\{\left(\mu_{1}^{\prime 2}-0.0901 z\right)^{2}+\mu_{1}^{\prime 2}\right\} \exp (-z)}{\left(6 \mu_{1}^{\prime 2}+0.8893\right)(1+\exp (-z))^{2}} ; z \in R, \alpha \in R
$$

Bounds for mean and variance: The following bounds for mean, variance can be derived using numerically optimizing $\mathrm{E}(Z)$ and $\operatorname{Var}(Z)$ with respect to $\alpha$.

$$
\begin{array}{ll}
\text { i. } & -1.29 \leq \mathrm{E}(Z) \leq 1.29 \text { and } \\
\text { ii. } & 3.29 \leq \operatorname{Var}(Z) \leq 13.82
\end{array}
$$

Plots of mean and variance: We have plotted the mean and the variance respectively in figure 4 and 5 to study their behavior. Theses plots also verify the bounds presented above.

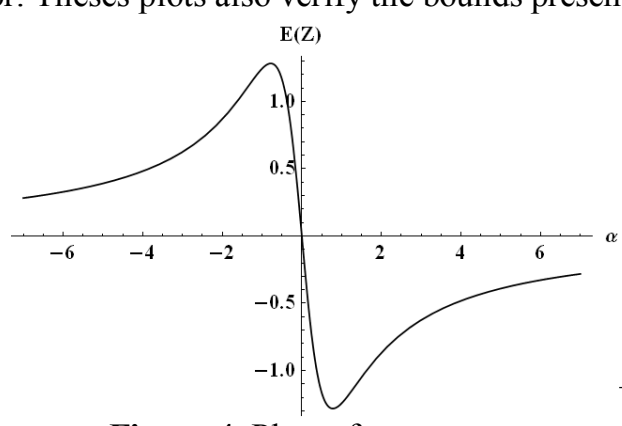

Figure 4. Plots of mean

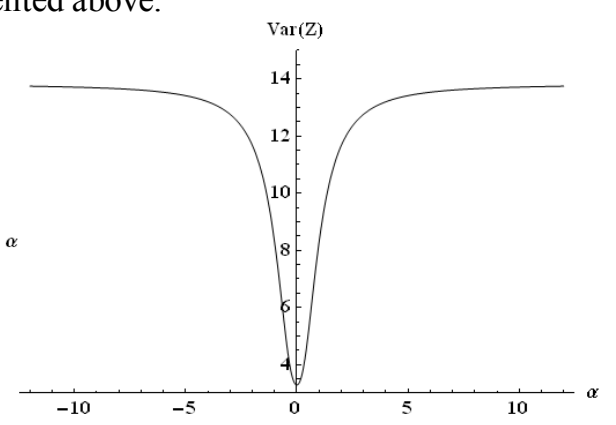

Figure 5 . Plots of variance

Remark 3. Moments of BLG Distribution can be derived easily by taking limit as $\alpha \rightarrow+\infty$ or $-\infty$ in the moments of $\operatorname{ASLG}(\alpha)$ in section 3.2.2 as

$$
\mathrm{E}(Z) \rightarrow 0, \quad \operatorname{Var}(Z) \rightarrow 7 \pi^{2} / 5=13.82
$$

\subsubsection{Skewness and Kurtosis}

The skewness and Kurtosis of $\operatorname{ASLG}(\alpha)$ is given by:

Skewness, $\gamma_{1}=\frac{\mu_{3}-3 \mu_{1} \mu_{2}+2 \mu_{1}^{3}}{\left(\mu_{2}-\mu_{1}^{2}\right)^{3 / 2}}=\frac{2 \sqrt{5} \alpha\left\{-45 C^{2}+90 C \pi^{2}+\alpha^{2}(-40+63 C) \pi^{4}\right\}}{\pi\left\{30 C+\alpha^{2}(-20+21 C) \pi^{2}\right\}^{3 / 2}}$

Kurtosis, $\gamma_{2}=\frac{\mu_{4}-4 \mu_{1} \mu_{3}+6 \mu_{1}^{2} \mu_{2}-3 \mu_{1}^{4}}{\left(\mu_{2}-\mu_{1}^{2}\right)^{2}}-3$

$$
=\frac{-21 \pi^{4} \alpha^{4}\{21 C(21 C-80)+800\}+45 \pi^{2} \alpha^{2} C\{C(465 C-1372)+1120\}+1890 C^{2}(7 C-10)}{7\left\{\pi^{2} \alpha^{2}(21 C-20)+30 C\right\}^{2}}
$$

where, $C=\left(6+\alpha^{2} \pi^{2}\right) / 3$. Note that, for $\alpha<(>) 0$ all odd order moments are positive (negative) while the even order moments are always positive.

Bounds for skewness and kurtosis: The following bounds for skewness and kurtosis can be derived using numerically optimizing $\gamma_{1}$ and $\gamma_{2}$ with respect to $\alpha$.

$$
\begin{array}{ll}
\text { i. } & -1.62 \leq \gamma_{1} \leq 1.62 \text { and } \\
\text { ii. } & -0.74 \leq \gamma_{2} \leq 2.17 .
\end{array}
$$

Plots of skewness and kurtosis: We have plotted the skewness and kurtosis respectively in figure 6 and 7 to study their behavior. Theses plots also verify the bounds presented above.

Remark 4. Skewness and Kurtosis of BLG Distribution can be derived easily by taking limit as $\alpha \rightarrow+\infty$ or $-\infty$ in the results of $\operatorname{ASLG}(\alpha)$ in section 3.2.3 as $\gamma_{1} \rightarrow 0, \gamma_{2} \rightarrow-0.74$ 


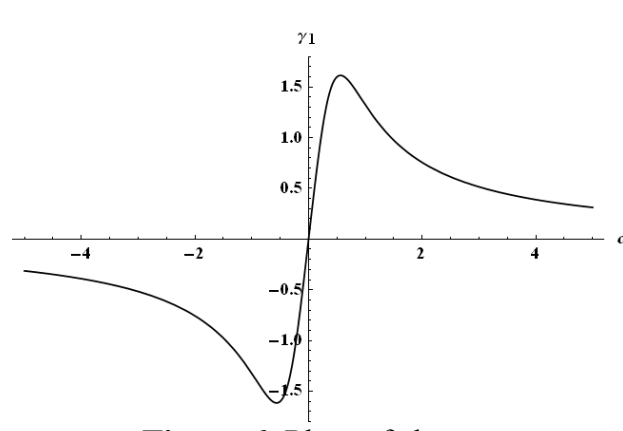

Figure 6. Plots of skewness

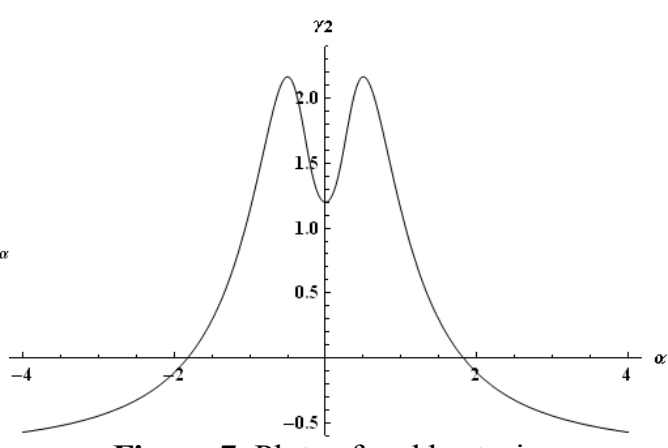

Figure 7. Plots of and kurtosis

\section{Truncated alpha-skew-logistic-distribution as a life time distribution}

Here we present alpha-Skew-logistic-distribution truncated below ' 0 ' as a potential life time distribution. The pdf of the truncated-alpha-logistic-distribution TASLG $(\alpha)$ truncated below ' 0 ' is given by

$$
f_{T}(t ; \alpha)=\frac{6\left\{(1-\alpha t)^{2}+1\right\} \exp (-t)}{(1+\exp (-t))^{2}\left(\alpha^{2} \pi^{2}-4.16 \alpha+6\right)} ; t>0
$$

provided, $\alpha^{2} \pi^{2}-4.16 \alpha+6>0$.

The survival function $S_{T}(t ; \alpha)$ and the hazard rate functions $h_{T}(t ; \alpha)$ of TASLG $(\alpha)$ can be easily expressed in terms of the pdf $f_{Z}(t ; \alpha)$ and cdf $F_{Z}(t, \alpha)$ of $\operatorname{ASLG}(\alpha)$ as

$$
S_{T}(t ; \alpha)=\frac{1-F_{Z}(t, \alpha)}{1-F_{Z}(0, \alpha)} \text { and } h_{T}(t ; \alpha)=\frac{f_{T}(t ; \alpha)}{S_{T}(t ; \alpha)}=\frac{f_{Z}(t ; \alpha)}{1-F_{Z}(t, \alpha)}
$$

For admissible values of the parameter $\alpha$, we have plotted the $h_{T}(t ; \alpha)$ in figure 8 to study its behavior graphically.
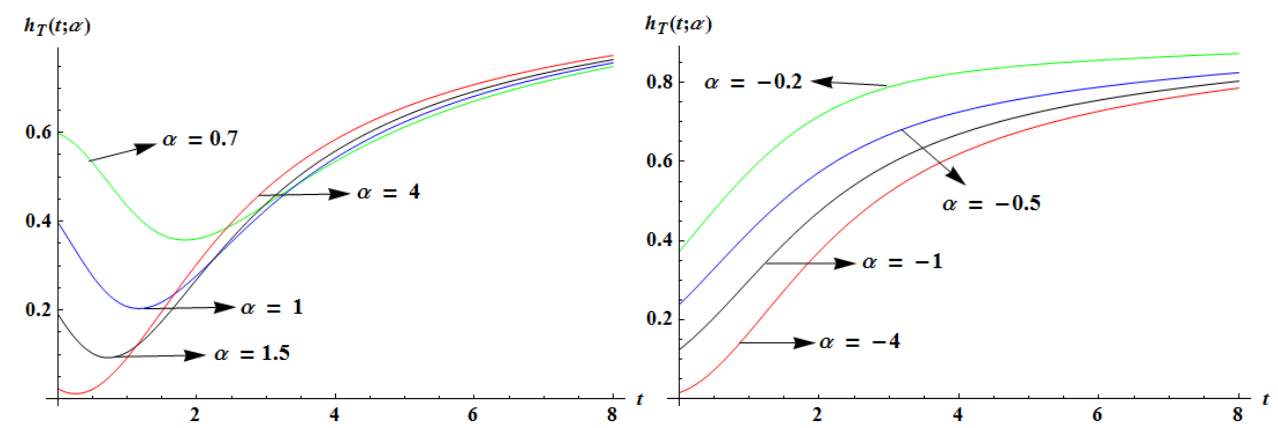

Figure 8. Plots of hazard rate function TASLG $(\alpha)$

From the figure 8 it can be seen that for $\alpha \leq 0$ the hazard rate is increasing always while for $\alpha>0.7$ it assumes bathtub shape. For values of $\alpha \in(0,0.7)$ the hazard rate takes different shapes. Thus depending on the range of the values of the parameter $\alpha$, the hazard rate function of TASLG $(\alpha)$ assumes different useful shapes like the Weibull model and thus has the potential to be a flexible life time model.

Remark 5. Clearly, for $\alpha=0$, TASLG $(\alpha)$ reduces to standard half logistic distribution (Balakrishnan, 1992). As such the present TASLG $(\alpha)$ may be seen as a generalization of the standard half logistic distribution (Balakrishnan, 1992).

\section{Closeness of alpha skew normal and alpha skew logistic distributions}

Similarities of normal and logistic have been examined in the statistical literature (Johnson et al. (2004), pp. 119,). Since the alpha skew logistic distribution proposed here has the same structure as that of the alpha skew normal distribution of Elal-Olivero (2010), we present results of a brief numerical investigation carried out to check their closeness. Accordingly, the difference of the pdfs of $\operatorname{ASN}(a, 0, \sigma)$ and $\operatorname{ASLG}(\alpha, 0, \beta)$, have been studied to find combinations of parameters such that the two pdfs almost coincide. This is achieved by minimizing the sum of the squared difference of the pdfs over a finite set of equally spaced points in the range of these two distributions with respect to one parameter while others are kept fixed. The KL distance 
(Burnham and Anderson, 2002) of these pairs are then checked to re-emphasize their closeness. We found many close pairs, two such close pairs are

I. $\operatorname{ASN}(2.5,0,2)$ and $\operatorname{ASLG}(1.656,0,1)$ :

Distance between the pdfs $=0.00265$ and $\mathrm{KL}$ distance $=0.03275$.

II. $\operatorname{ASN}(3.8535,0,6)$ and $\operatorname{ASLG}(2.5,0,3)$ :

Distance between the pdfs $=0.00896$ and $\mathrm{KL}$ distance $=0.03572$.

We have presented the graphs of the pdfs, cdfs and difference of cdfs of the first pair of distributions respectively in Figure 9, 10 and 11 to depict their closeness.

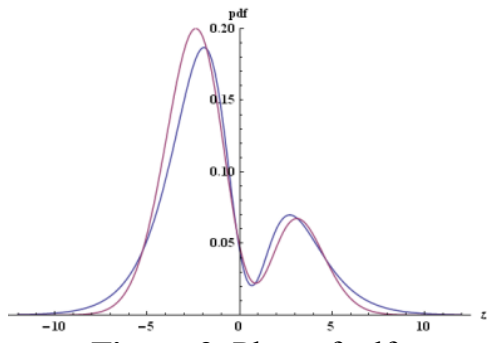

Figure 9. Plots of pdf

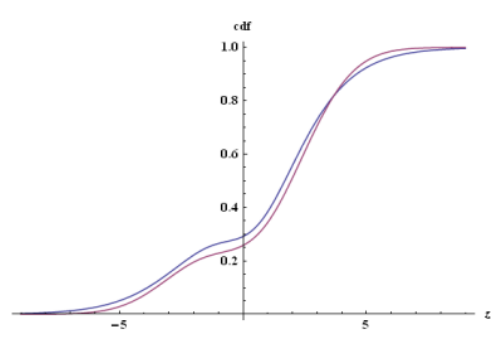

Figure 10. Plots of cdf

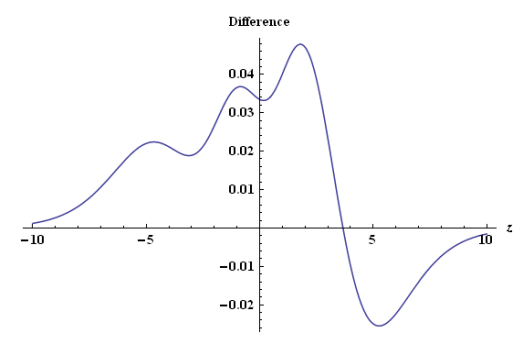

Figure 11. Plots of differences of cdf

\section{Parameter estimation}

We present the problem of parameter estimation of a location and scale extension of ASLG $(\alpha)$.

If $Z \sim \operatorname{ASLG}(\alpha)$, then $Y=\mu+\beta Z$ is said to be the location $(\mu)$ and scale extension $(\beta)$ of $Z$ and has the pdf

$$
f_{Y}(y ; \alpha, \mu, \beta)=\frac{3\left\{(1-\alpha(y-\mu) / \beta)^{2}+1\right\} \exp (-(y-\mu) / \beta)}{\left(6+\alpha^{2} \pi^{2}\right) \beta(1+\exp (-(y-\mu) / \beta))^{2}} ; y \in R, \alpha, \mu \in R, \beta>0
$$

We denote it by $Y \sim \operatorname{ASLG}(\alpha, \mu, \beta)$.

In particular, for $\alpha \rightarrow+\infty$ or $-\infty, \operatorname{ASLG}(\alpha, \mu, \beta) \rightarrow$ BLG .

\subsection{Method of moments}

Let $m_{1}, m_{2}$ and $m_{3}$ are respectively the first three sample raw moments for a given random sample $\left(y_{1}, y_{2}, \cdots, y_{n}\right)$ of size $n$ drawn from $\operatorname{ASLG}(\alpha, \mu, \beta)$ distribution in equ. (12). Then the moment estimates of the three parameters $\mu, \sigma$ and $\alpha$ are obtained by simultaneously solving the following equations derived by equating first three population moments with corresponding sample moments.

$$
\begin{aligned}
& m_{1}=\mu-\frac{2 \sigma \alpha \pi^{2}}{6+\alpha^{2} \pi^{2}} \Rightarrow \mu=\frac{2 \sigma \alpha \pi^{2}}{6+\alpha^{2} \pi^{2}}+m_{1} \\
& m_{2}=\mu^{2}+\frac{-20 \alpha \pi^{2} \mu \sigma+\pi^{2}\left(10 \pi^{2}+7 \alpha^{2} \pi^{2}\right) \sigma^{2}}{5\left(6+\alpha^{2} \pi^{2}\right)} \\
& m_{3}=\mu^{3}+\frac{-30 \alpha \pi^{2} \mu^{2} \sigma+3 \pi^{2}\left(10+7 \alpha^{2} \pi^{2}\right) \mu \sigma^{2}-14 \alpha \pi^{4} \sigma^{3}}{5\left(6+\alpha^{2} \pi^{2}\right)}
\end{aligned}
$$

Substituting the value of $\mu$ from equ. (13) in equ. (14) and solving for $\sigma^{2}$ we get,

$$
\sigma^{2}=\frac{5\left(m_{2}-m_{1}^{2}\right)\left(6+\alpha^{2} \pi^{2}\right)^{2}}{\pi^{2}\left(60+32 \alpha^{2} \pi^{2}+7 \alpha^{4} \pi^{4}\right)}
$$

Finally, putting these values of $\mu$ and $\sigma^{2}$ in equ. (15), we get the following equ. in $\alpha$

$$
m_{3}=m_{1}{ }^{3}+3 m_{1}\left(m_{2}-m_{1}{ }^{2}\right)+\frac{4 \sqrt{5} \alpha \pi^{4}\left(-36+16 \alpha^{2} \pi^{2}+7 \alpha^{4} \pi^{4}\right)}{\left(6+\alpha^{2} \pi^{2}\right)^{3}}\left(\frac{\left(6+\alpha^{2} \pi^{2}\right)^{2}\left(m_{2}-m_{1}{ }^{2}\right)}{60+32 \alpha^{2} \pi^{2}+7 \alpha^{4} \pi^{4}}\right)^{3 / 2}
$$


While the exact solution of the equ. (17) is not tractable, it can be numerically solved to estimate $\alpha$. Once $\alpha$ is estimated rest of the two parameters namely, $\mu$ and $\sigma$ can be directly estimated from equ. (13) and (16) respectively.

Remark 6. It may be noted that the equ. (17) may return multiple solutions for $\alpha$ and hence leading to more than one set of feasible estimates for the parameters. This situation may be resolved by retaining the best set (according to some criterion e.g., likelihood or entropy) among the feasible solutions.

\subsection{Maximum likelihood estimation}

For a random sample of size $n\left(y_{1}, y_{2}, \cdots, y_{n}\right)$ drawn from $\operatorname{ASLG}(\alpha, \mu, \beta)$ distribution in equ. (12), the likelihood function is given by

$$
\begin{aligned}
\log L= & \log \left[\prod_{i=1}^{n} f_{Y}(y ; \alpha, \mu, \beta)\right] \\
= & \frac{-n \beta\left(6+\alpha^{2} \pi^{2}\right)}{3}+\sum_{i=1}^{n}\left(\log \left[\left(1-\alpha\left(y_{i}-\mu\right) / \beta\right)^{2}+1\right]-2 \log \left[\left(1+\exp \left(-\left(y_{i}-\mu\right) / \beta\right)\right)\right]\right) \\
& -\sum_{i=1}^{n}\left(y_{i}-\mu\right) / \beta
\end{aligned}
$$

The MLE's of $\mu, \beta$ and $\alpha$ are obtained by numerically maximizing $\log L$ with respect to $\mu, \beta$ and $\alpha$. MLEs as,

The variance-covariance matrix of the MLEs can be derived by using the asymptotic distribution of

$$
\left[\begin{array}{lll}
\mathrm{E}\left(\frac{-\partial^{2} \log L}{\partial \mu^{2}}\right) & \mathrm{E}\left(\frac{-\partial^{2} \log L}{\partial \mu \partial \beta}\right) & \mathrm{E}\left(\frac{-\partial^{2} \log L}{\partial \mu \partial \alpha}\right) \\
\mathrm{E}\left(\frac{-\partial^{2} \log L}{\partial \beta \partial \mu}\right) & \mathrm{E}\left(\frac{-\partial^{2} \log L}{\partial \beta^{2}}\right) & \mathrm{E}\left(\frac{-\partial^{2} \log L}{\partial \beta \partial \alpha}\right) \\
\mathrm{E}\left(\frac{-\partial^{2} \log L}{\partial \alpha \partial \mu}\right) & \mathrm{E}\left(\frac{-\partial^{2} \log L}{\partial \alpha \partial \beta}\right) & \mathrm{E}\left(\frac{-\partial^{2} \log L}{\partial \alpha^{2}}\right)
\end{array}\right]^{-1}
$$

When the exact expressions for various expectations above are cumbersome, in practice they are estimated as

$$
\mathrm{E}\left(\frac{-\partial^{2} \log L}{\partial \mu^{2}}\right) \approx-\left.\frac{\partial^{2} \log L}{\partial \mu^{2}}\right|_{\mu=\hat{\mu}},\left.\mathrm{E}\left(\frac{-\partial^{2} \log L}{\partial \mu \partial \beta}\right) \approx \frac{-\partial^{2} \log L}{\partial \mu \partial \beta}\right|_{\mu=\hat{\mu}, \alpha=\hat{\alpha}} \text { etc. }
$$

\section{Real life applications: comparative data fitting}

Here we have considered data set of the heights of 100 Australian athletes (Cook and Weisberg, 1994) and compared the proposed distribution $\operatorname{ASLG}(\alpha, \mu, \beta)$ with the logistic distribution $L(\mu, \beta)$, skew logistic distribution $S L(\lambda, \mu, \beta)$ of Wahed and Ali (2001), the new skew logistic distribution $L_{S}(\lambda, \alpha, \beta, \mu)$ of Chakraborty et al. (2012), alpha-skew-normal distribution $\operatorname{ASN}(\alpha, \mu, \beta)$ of Elal-Olivero (2010), alpha-skewLaplace distribution $\operatorname{ASL}(\alpha, \mu, \beta)$ of Harandi and Alamatsaz (2013) and alpha skew normal slash distribution $\operatorname{ASNS}(\alpha, q, \mu, \beta)$ of Gui (2014). The MLE of the parameters are obtained by using numerical optimization routine. In the present work, the GenSA-package, Version-1.0.3, of the software $R$ is used. Akaike Information Criterion (AIC) and Beysian Information Criterion (BIC) are used for model comparison.

Further, since $\mathrm{L}(\mu, \beta)$ and $\operatorname{ASLG}(\alpha, \mu, \beta)$ are nested models the likelihood ratio (LR) test is used to discriminate between them.

The LR test is carried out to test the following hypothesis

against the alternative

$$
H_{0}: \alpha=0 \text {, that is the sample is drawn from } L(\mu, \beta),
$$

$$
H_{1}: \alpha \neq 0 \text {, that is the sample is drawn from } \operatorname{ASLG}(\alpha, \mu, \beta) .
$$

From the Table 1 it is seen that the proposed alpha skew logistic distribution $\operatorname{ASLG}(\alpha, \mu, \beta)$ provides best fit to the data set under consideration in terms of all the criteria, namely the log-likelihood, the AIC as well 
as the BIC. The plots of observed (in histogram) and expected densities (lines) presented in Figure 9, also confirms our finding.

LR test result: The value of LR test statistic is 5.058 which exceed the $95 \%$ critical value, 3.84 . Thus there is evidence in favors of the alternative hypothesis that the sampled data comes from $\operatorname{ASLG}(\alpha, \mu, \beta)$ not from $\mathrm{L}(\mu, \beta)$.

Table 1: MLE's, log-likelihood, AIC and BIC for heights (in cm) of 100 Australian Athletes data

\begin{tabular}{|c|c|c|c|c|c|c|c|c|}
\hline $\begin{array}{c}\text { Parameters } \rightarrow \\
\text { Distributions } \downarrow\end{array}$ & $\mu$ & $\beta$ & $\lambda$ & $\alpha$ & $q$ & $\begin{array}{c}\text { Log- } \\
\text { likelihood }\end{array}$ & AIC & BIC \\
\hline$L(\mu, \beta)$ & 175.001 & 4.410 & -- & -- & -- & -349.589 & 703.179 & 708.388 \\
\hline$S L(\lambda, \mu, \beta)$ & 179.563 & 5.197 & -0.901 & -- & -- & -348.689 & 703.378 & 711.194 \\
\hline$L_{S}(\lambda, \alpha, \beta, \mu)$ & 172.909 & 4.620 & 1.646 & 2.037 & -- & -347.546 & 703.092 & 713.513 \\
\hline $\operatorname{ASN}(\alpha, \mu, \beta)$ & 169.465 & 7.280 & -- & -1.112 & -- & -348.000 & 701.994 & 709.810 \\
\hline $\operatorname{ASL}(\alpha, \mu, \beta)$ & 171.400 & 3.301 & -- & -1.091 & -- & -347.933 & 701.866 & 709.682 \\
\hline $\operatorname{ASNS}(\alpha, q, \mu, \beta)$ & 178.01 & 4.744 & -- & 0.550 & 2.548 & -349.552 & 707.104 & 717.525 \\
\hline $\operatorname{ASLG}(\alpha, \mu, \beta)$ & 170.496 & 3.203 & -- & -0.747 & -- & $\mathbf{- 3 4 7 . 0 6 0}$ & $\mathbf{7 0 0 . 1 2 1}$ & $\mathbf{7 0 7 . 9 3 6}$ \\
\hline
\end{tabular}

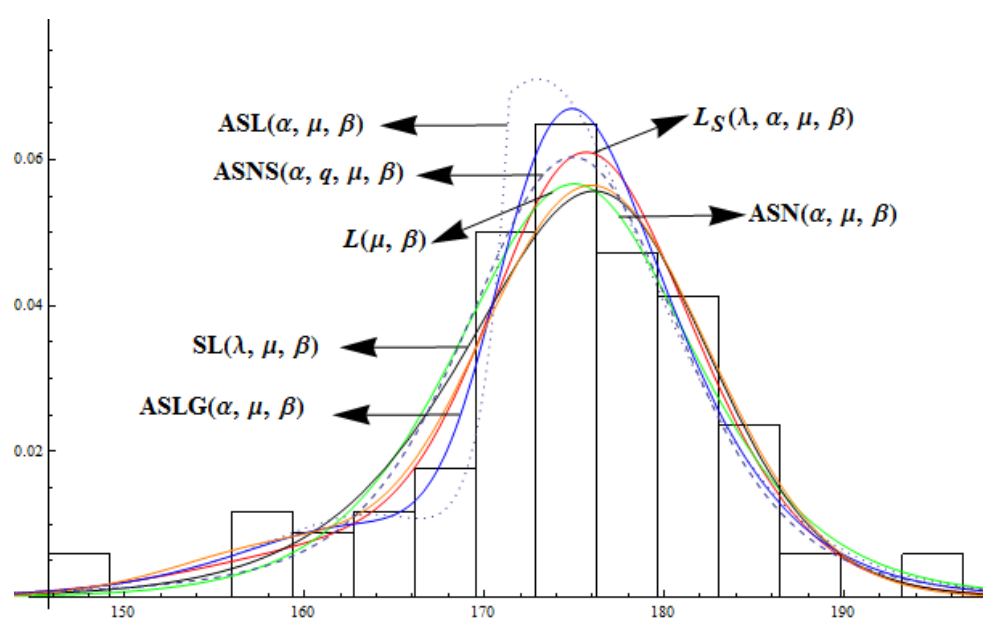

Figure 12. Plots of observed and expected densities for heights of 100 Australian athletes

Remark 7. The variance-covariance matrix of the MLEs of the parameters of $\operatorname{ASLG}(\alpha, \mu, \beta)$ is obtained by using (19) as

$$
\text { Variace-Covrance }(\hat{\alpha}, \hat{\mu}, \hat{\beta})=\left[\begin{array}{ccc}
0.6841 & -0.0646 & 0.0497 \\
-0.0646 & 0.0921 & 0.0209 \\
0.0497 & 0.0209 & 0.0240
\end{array}\right]
$$

\section{Concluding remarks}

In this article a new alpha skew logistic distribution which includes both unimodal as well as bimodal logistic distributions is introduced and some of its basic properties are investigated. A truncated version of the proposed distribution is seen to have bathtub shaped failure rate function. Closeness of the alpha skew normal and alpha skew logistic distributions is briefly explored.

The numerical results of the modeling of a real life data set considered here has shown that the proposed distribution provides better fitting in comparison to the logistic and skew logistic distribution of Wahed and Ali (2001), alpha-skew-normal distribution $\operatorname{ASN}(\alpha, \mu, \beta)$ of Elal-Olivero (2010), alpha skew logistic distribution $\operatorname{ASL}(\alpha, \mu, \beta)$ of Harandi and Alamatsaz (2013), the new multimodal skew logistic distribution of Chakraborty et al. (2012) and alpha skew normal slash distribution of Gui (2014). 


\section{References}

[1] Azzalini, A. (1985) A class of distributions which includes the normal ones. Scand. J. Stat. 12: 171 - 178.

[2] Balakrishnan, N. (1992) Handbook of Logistic Distribution. Marcel Dekker. New York.

[3] Burnham, K. P., Anderson, D. R. (2002) Model Selection and Multimodel Inference: A Practical Information Theoretic Approach. $2^{\text {nd }}$ edn. Springer.New York.

[4] Chakraborty, S., Hazarika, P. J. (2011) A survey of the theoretical developments in univariate skew normal distributions. Assam Statist. Rev. 25(1): $41-63$.

[5] Chakraborty, S. Hazarika, P. J., Ali, M. M. (2012) A new skew logistic distribution and its properties. Pak. J. Statist. 28(4): 513 524.

[6] Chakraborty, S. Hazarika, P. J., Ali, M. M. (2014a) A multimodal skewed extension of normal distribution: its properties and applications. Statistics-A Journal of Theoretical and Applied Statistics, http://dx.doi.org/10.1080/ 02331888.2014.908880.

[7] Chakraborty, S. Hazarika, P. J., Ali, M. M. (2014b) A multimodal skew-Laplace distribution: its properties and applications. Pak. J. Statist, 30(2): 253-264.

[8] Cook, R. D., Weisberg, S. (1994) An Introduction to Regression Analysis. Wiley. New York.

[9] Elal-Olivero, D. (2010) Alpha-skew-normal distribution. Proyecciones Journal of Mathematics. 29: 224 - 240.

[10] Gui, W. (2014) A generalization of the slashed distribution via alpha skew normal distribution. Stat Methods Appl. DOI 10.1007/s10260-014-0258-7.

[11] Harandi, S. S., Alamatsaz, M. H. (2013) Alpha-skew-Laplace distribution, Statist Probab Lett. 83(3):774 - 782.

[12] Huang, W. J., Chen, Y. H. (2007) Generalized skew Cauchy distribution, Statist Probab Lett. 77:1137 - 1147.

[13] Johnson, N. L., Kotz, S., Balakrishnan, N. (2004) Continuous Univariate Distributions (Volume 2). A Wiley-Interscience Publication. John Wiley \& Sons Inc. New York.

[14] Nadarajah, S. (2009) The skew logistic distribution. AStA Adv Stat Anal. 93: 187 - 203.

[15] Nadarajah, S., Kotz, S. (2006) Skew distributions generated from different families. Acta Appl Math. 91:1 - 37.

[16] Nadarajah, S., Kotz, S. (2007) Skew models I. Acta Appl Math. 98:1 - 28.

[17] Prudnikov, A. P., Brychkov, Y. A. and Marichev, Y. I. (1986) Integrals and Series, (Volume - 1), Gordon and Breach. Amsterdam.

[18] Wahed, A. S., Ali, M. M. (2001) The skew logistic distribution. J Stat. Res. 35:71 - 80. 